\title{
Artefacts : nature, structure et usages
}

\section{Artefacts: nature, structure and usages}

Michel Feugère ${ }^{1}$, Amélie Aude Berthon $^{2}$, Hervé Bohbot ${ }^{3}$, Alexis Bonnefoy ${ }^{4}$, Yves Bourrieau ${ }^{5}$, Maxime Callewaert ${ }^{6}$, Antony Carbone ${ }^{7}$, Lucile Catté ${ }^{8}$, Patrick Defaix ${ }^{9}$, Louis Eyango ${ }^{10}$, Amaury Gilles ${ }^{11}$, Alyssa Giraudo ${ }^{12}$, Chloé Landrieux ${ }^{13}$, Patrick Mosca ${ }^{14}$, Marie-Pauline Pringalle $^{15}$, Jean Soulat ${ }^{16}$, Clément Tournier ${ }^{17}$, Elise Vigier ${ }^{18}$, Bénédicte Viroulet ${ }^{19}$

\footnotetext{
${ }^{1}$ chercheur CNRS, Univ. Lyon, UMR 5138 (ArAr), 7 rue Raulin, 69007 Lyon ; michel.feugere@mom.fr

${ }^{2}$ spécialiste Éveha, 44 rue Proudhon, 63000 Clermont-Ferrand, UMR 5138 (ArAr) ; amelie.berthon@eveha.fr

3 ingénieur CNRS, Univ. Paul-Valéry Montpellier, UMR 5140 (ASM), route de Mende, 34199 Montpellier Cedex 5 ;

herve.bohbot@cnrs.fr

${ }^{4}$ chercheur associé, Univ. Lyon, UMR 5138 (ArAr), 7 rue Raulin, 69007 Lyon ; alexis.bonnefoy@gmail.com

${ }^{5}$ coll. bénévole ; yves.bourrieau@gmail.com

${ }^{6}$ Musée Royal de Mariemont, Chaussée de Mariemont 100, 4140 Morlanweitz (BE) ; mcallewaert@gmail.com

${ }^{7}$ doctorant, Univ. Lyon, UMR 5138 (ArAr), 7 rue Raulin, 69007 Lyon ; carbone.antony@gmail.com

${ }^{8}$ chercheuse associée, Univ. Lyon, UMR 5138 (ArAr), 7 rue Raulin, 69007 Lyon ; lucile.catte@gmail.com

9 coll. bénévole ; defaix.patrick@orange.fr

${ }^{10}$ analyste-programmeur, Univ. Lyon, UMR 5138, 7 rue Raulin, 69007 Lyon ; charly.eyango@gmail.com

${ }^{11}$ docteur, Univ. Lyon, UMR 5138 (ArAr), 7 rue Raulin, 69007 Lyon ; amaury.gilles@gmail.com

12 masterante, Univ. Lyon, UMR 5138 (ArAr), 7 rue Raulin, 69007 Lyon ; Alyssa.Giraudo@hotmail.fr

${ }^{13}$ étudiante, Univ. Lyon, UMR 5138 (ArAr), 7 rue Raulin, 69007 Lyon ; chloe.landrieux@gmail.com

14 coll. bénévole ; moscapat@orange.fr

${ }^{15}$ coll. bénévole ; pauline.pringalle@gmail.com

${ }^{16}$ spécialiste Laboratoire Landarc (UMR 6273 Craham) ; jean.soulat@landarc.fr

17 archéologue, Archeodunum SAS ; c.tournier@archeodunum.fr

${ }^{18}$ doctorante Univ. Lyon, UMR 5138 (ArAr), 7 rue Raulin,69007 Lyon ; vigier.elise1988@gmail.com

${ }^{19}$ Conservatrice, Musée Gallo-Romain, Place de l’Eglise, 68600 Biesheim ; b.viroulet@biesheim.fr
}

RÉSUMÉ. Cet article se propose de présenter Artefacts, l'encyclopédie collaborative en ligne des objets archéologiques, qui tente de recenser l'ensemble des formes d'objets attestées entre la fin de la Préhistoire et la période protoindustrielle, en Europe et sur le pourtour méditerranéen. On aborde successivement les principes du projet, l'historique de son développement ainsi que l'architecture générale du site web. Sont évoqués ensuite les publics ciblés et leurs usages respectifs de ce projet collaboratif, en particulier les fonctionnalités spécifiquement destinées aux chercheurs.

ABSTRACT. This article aims at describing "Artefacts, online collaborative encyclopaedia of archaeological small finds", which tries to list all the forms of objects attested between the end of the Prehistory and the pre-industrial period in Europe and around the Mediterranean. It successively discusses the principles of the project, the history of its development as well as the general architecture of the website. The target audience and its respective practices of this collaborative project, in particular, are then evoked as well as the features specifically intended for researchers.

MOTS-CLÉS. humanités numériques, base de données, encyclopédie, objets archéologiques, typologie, recherche. KEYWORDS. digital humanities, database, encyclopaedia, archaeological small finds, typology, research.

On trouvera dans cet article les éléments fondamentaux sur la nature, l'organisation structurelle et l'utilisation du site internet Artefacts.mom.fr.

\section{Principe}

Artefacts (figure 1) est essentiellement une base de données consacrée aux objets mobiliers archéologiques, à l'exclusion de la vaisselle en céramique et des monnaies. Il s'agit à la fois d'un outil documentaire et d'un outil de recherche, proposant à chaque utilisateur des fonctionnalités variables et croissantes en fonction de son investissement personnel dans le projet. Le champ couvert est très vaste puisqu'il va de la fin de la préhistoire à l'époque contemporaine, comprenant l'âge du Bronze, les âges 
du Fer, l'Antiquité romaine, les périodes médiévale, moderne et contemporaine jusqu'au début du XX $\mathrm{s}$. Toutes les périodes ne sont pas traitées de manière aussi approfondie puisque le contenu est fonction du travail apporté par les auteurs qui enregistrent des données. Malgré tout, ce sont aujourd'hui plus de 300000 objets qui sont réunis sur près de 20000 fiches typologiques.

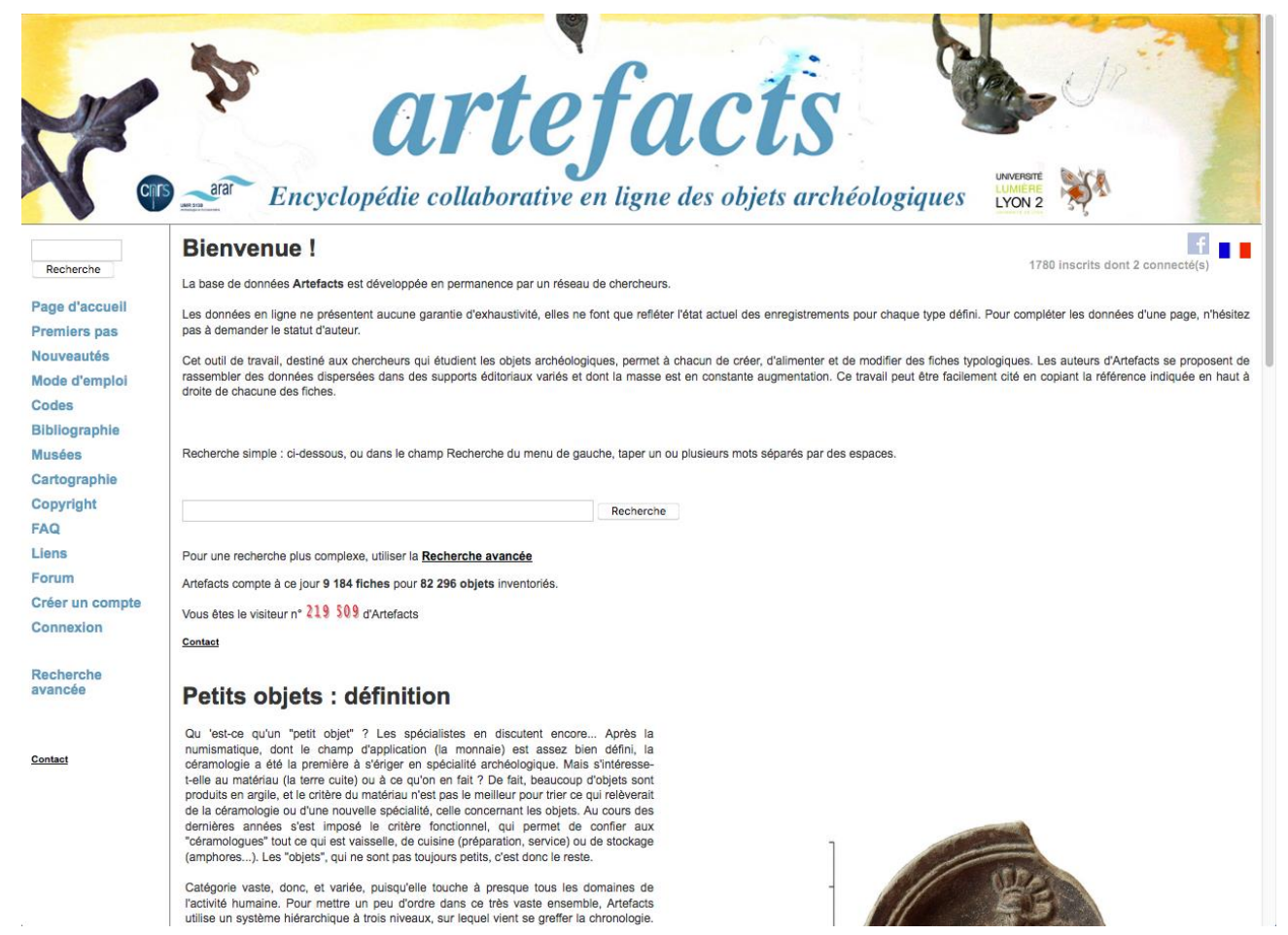

Figure 1. Page d'accueil d'artefacts.mom.fr

Quelques chiffres au 15/07/18 :

- 121054 objets sur 19132 fiches.

- 26425 sites géoréférencés.

- 1093 musées dans 39 pays.

- 20984 titres bibliographiques, dont 36,58\% accessibles en ligne.

La base de données est alimentée actuellement par environ 150 auteurs, principalement français mais également originaires d'autres pays européens, qui se répartissent entre étudiants, archéologues de terrain, chercheurs, conservateurs de musées et simples bénévoles. D'après le profil des visiteurs les plus assidus, Artefacts est d'abord utilisé par les archéologues du secteur préventif, notamment pour l'identification des découvertes qui doivent être décrites dans les rapports de fouilles. L'autre catégorie de visiteurs la plus motivée est celle des conservateurs et personnels des musées, pour le classement des collections locales, et les étudiants dont les recherches personnelles concernent les catégories traitées dans Artefacts.

La fonctionnalité la plus appréciée du site est la bibliographie, souvent la porte d'entrée des visiteurs qui découvrent ensuite les contenus et autres outils proposés par Artefacts (figure 2). Dans les fiches typologiques, la cartographie automatique est également très prisée pour son côté dynamique, les cartes se mettant à jour au fil des apports. 
Bermond 1998: I. Bermond, L'occupation protohistorique au Nord-Est du Bassin de Thau (région de Mèze, Hérault). In : S. Mauné (dir.), Recherches récentes sur les établissements ruraux protohistoriques en Gaule méridionale (IXe - Ille s. av. J.-C.). Actes de la table-ronde de Lattes (mai 1997) (Protohistoire Européenne, 2), Montagnac 1998, 29-43.

Bessac 2004 : J.-Cl. Bessac, Le tournage antique deéléments architecturaux. In : M. Feugère, J.-C. Gérold (dir.), Le tournage, des origines à l'an Mil. Actes du colloque de Niederbronn, octobre 2003 (Monogr. Instrumentum, 27), Montagnac 2004, 187-200. ()

Besson 2015 : C. Besson, Un autre regard sur la bijouterie en or de Lyon (Rhône). In: St. Raux, I. Bertrand, M. Feugère (dir.), Actualité de la recherche sur les mobiliers non céramiques de "Antiquité et du haut Moyen Age. Actes de la table-ronde européenne Instrumentum, Lyon (F, Rhône), 18-20 octobre 2012 (Monogr. Instrumentum, 51) Montagnac/ Chauvigny 2015, 555-576.

Bet, Dejage 1996 : Ph. Bet, R. Delage, Principes généraux de gestion du mobilier archéologique : une étape primordiale dans linformatisation des données sur le site de Lezoux : actes du (1) 11 congrès de Dijon, SFECAG, 1996, 263-286.

Binet, Hoët-van Cauwenberghe 2010 : E. Binet, Chr. Hoët-van Cauwenberghe, Graffiti sur éléments architecturaux à Samarobriva (Amiens). Revue du Nord 92, 2010, $n^{\circ} 388,203-224$.

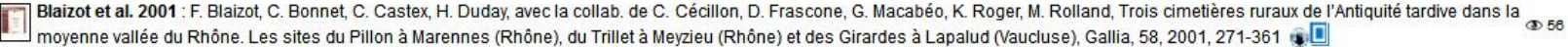

Blaizot et al. 2008 : F. Blaizot, S. Raux, C. Bonnet, E. Henry, V. Forest, P. Ecard, C. Jorda, G. Macabéo, L'ensemble funéraire rural de Malbosc (Montpellier, Hérault) : pratiques funéraires de 41 I'Antiquité tardive, RAN, 41, 2008, 53-152

Blanchard et al. 2015 : P. Blanchard, A. Baron, D. Henri, H. Réveillas, S. Kacki, D. Castex, R. Giuliani, Vestiges mobiliers associés aux défunts du secteur central de la catacombe des saints Pierre et Marcellin à Rome (ler - Ille s. ap. J.-C.). In: St. Raux, I. Bertrand, M. Feugère (dir.). Actualité de la recherche sur les mobiliers non céramiques de l'Antiquité et du haut Moyen Age. Actes de la table-ronde européenne Instrumentum, Lyon (F, Rhône), 18-20 octobre 2012 (Monogr. Instrumentum, 51) Montagnac/ Chauvigny 2015, 245-267. F

Boucher, Lusson 2018 : Th. Boucher, D. Lusson, coll. B. Robin, L'instrumentum. In: F. Couvin (dir.), Deux établissements ruraux laténiens et gallo-romains du plateau de la Petite Beauce: "Beaudisson" et "la Gueule" II à Mer (Loir-et-Cher) (Suppl. 68 à la RACF), Tours 2018, 188-233. [0

Brissaud, Loiseau 2015: L. Brissaud, Chr. Loiseau, Les canalisations en bois: techniques de mise en œuvre, diffusion, chronologie en Gaule romaine, étude de cas. In: St. Raux, I. Bertrand M. Feugère (dir.), Actualité de la recherche sur les mobiliers non céramiques de l'Antiquité et du haut Moyen Age. Actes de la table-ronde européenne Instrumentum, Lyon ( $F$, Rhône), 18-20 octobre 2012 (Monogr. Instrumentum, 51) Montagnac/ Chauvigny 2015, 487-516. 프.

Brives, Dumas-Lattaque 2015 : A.L. Brives, P. Dumas-Lattaque, L'apport du petit mobilier à la compréhension de létablissement rural du "Champ Drillon" à Bézannes (Marne). In: St. Raux, I. Bertrand, M. Feugère (dir.), Actualité de la recherche sur les mobiliers non céramiques de l'Antiquité et du haut Moyen Age. Actes de la table-ronde européenne Instrumentum, Lyon ( $F$, Rhône)

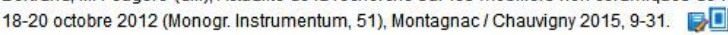

Figure 2. Aperçu du module Bibliographie. Les flèches pointées vers le bas indiquent qu'une version en ligne est disponible. Les rectangles bleus renvoient aux fiches typologiques comportant des objets de cette publication. Le symbole € fournit un lien vers le site de l'éditeur. La page associée à un crayon signale un compte-rendu en ligne.

Artefacts comporte une partie publique, qui représente la totalité des listes de musées et la bibliographie ; au sein de cette section publique, l'accès détaillé à la moitié environ des fiches typologiques se fait sur simple inscription en ligne, ce qui permet de bloquer les bots malveillants. Le statut de "membre inscrit" permet l'enregistrement de nouvelles références bibliographiques et l'accès à un forum de discussion, mais sans autre possibilité d'intervention sur le contenu du site. À ce jour (15/07/18), plus de 1700 personnes se sont inscrites en ligne pour bénéficier de ces fonctions, et environ 150 d'entre elles ont souhaité accéder au statut d'auteur qui donne des droits plus étendus.

Ce statut est réservé aux personnes susceptibles d'apporter des précisions au contenu d'Artefacts. Il s'acquiert en proposant aux administrateurs du site un projet impliquant une participation plus active au contenu du site (alimenter une catégorie de mobilier, enregistrer les données d'un site ou d'un musée, classer une série morpho-typologique, etc.).

L'utilisation d'Artefacts comme base de données documentaire permet d'accéder à des séries de contenus sur des recherches répondant à des questions très diverses : forme d'objet, provenance, type, auteur ou encore sujet décoratif... Les retours indiquent que beaucoup de « curieux » utilisent le site pour connaître par exemple les objets dont le décor fait appel à tel ou tel dieu romain, ou encore les formes de haches avec leur usage, ou les types de parures d'une période donnée... Pour cette catégorie d'utilisateurs, la seule sélection de fiches en ligne répond largement aux besoins exprimés.

La catégorie des auteurs se rattache, d'une manière ou d'une autre, au monde de la recherche. Il s'agit là d'utilisateurs qui disposent de données à classer et/ou de connaissances parfois très spécialisées. Après avoir utilisé le site comme simples visiteurs, ces utilisateurs motivés manifestent leur souhait d'accéder au statut d'auteur qui permet de visualiser toutes les fiches typologiques et d'intervenir sur le contenu des pages.

Les différentes interventions d'un auteur sur le site sont mémorisées, de manière à pouvoir les retrouver et en corriger éventuellement le contenu. Le logo de l'organisme d'appartenance, si l'auteur en a choisi un, est affiché automatiquement au bas de chaque page modifiée. Chaque auteur a 
également la possibilité de signer une fiche, s'il considère que son intervention constitue une contribution notable à son contenu. Il choisit alors de s'afficher comme auteur principal ou comme ou simple collaborateur de cette fiche (donnée publique).

\section{Historique}

Artefacts est l'héritier de projets nés au cours de la deuxième moitié des années 1980 dans le cadre de Syslat ${ }^{\circledR}$, système de documentation et d'étude des données de fouilles élaboré sur le site de Lattes (Hérault) par M. Py et son équipe. La mise en place de Syslat ${ }^{\circledR}$ a d'abord consisté, à partir d'un processus d'enregistrement des données de fouilles basé sur le système de Winchester (M. Bats et al., 1986), à construire un archipel documentaire permettant à des chercheurs parfois éloignés du terrain de travailler sur des fichiers reliés à une base commune (M. Py et al., 1991 ; 1997).

Assez vite, la description des types de mobiliers dans Syslat ${ }^{\boxplus}$ a montré la nécessité de bases de référence. Il s'agissait alors de construire des répertoires locaux, intégrés à l'interface de saisie ; le premier à être élaboré fut naturellement celui des formes céramiques, publié peu après sous la forme d'un volume papier (M. Py, 1993). Vinrent ensuite les monnaies, d'abord regroupées sous la forme d'un catalogue lattois appuyé sur une base de référence extérieure (M. Py, 2006), puis comme un véritable dictionnaire élargi à tous les types monétaires rencontrés dans une région plus étendue $(\mathrm{M}$. Feugère, M. Py, 2012).

Le principe de ces dictionnaires de référence — « répertorier tous les types attestés dans une région donnée »- se heurtait à chaque fois à la définition du territoire en question. Au départ, l'idée du projet était de ne prendre en compte que la typologie du mobilier de la fouille de Lattes. Un tel choix supposait déjà, pour la céramique, d'intégrer les classements de toutes les céramiques préromaines et romaines, locales et importées (étrusque, grecque, indigène, sigillée, amphore, etc). Une tâche gigantesque donc, dès le départ, que l'élargissement géographique n'aggravait pas trop fortement : on ne peut répertorier dans le département de l'Hérault, par exemple, qu'un nombre de types à peine supérieur à ce qui apparaît à Lattes. Pour les monnaies gauloises, le nombre d'émissions locales, qui peuvent parfois voyager très loin, imposait un territoire plus large : ce fut une petite moitié de la France, au Sud d'une ligne Angoulême-Chambéry.

Pour les objets, cette limite territoriale avait encore moins de sens. Elle ne présentait pas même l'avantage de la commodité puisqu'il n'y avait aucune raison de défavoriser les zones limitrophes. La surface de ce type de territoire ne peut être définie que par rapport à un sujet d'étude. Or les objets archéologiques peuvent faire l'objet d'importations sur des centaines, et parfois des milliers de kilomètres.

C'est la première raison pour laquelle Artefacts, qui n'était au départ qu'un miroir internet du dictionnaire de Syslat ${ }^{\circledR}$ consacré aux objets, Dicobj, en vint à prendre une autonomie croissante. L'aire géographique fut d'emblée très englobante, compte tenu de l'espace au sein duquel les objets circulent dans l'Antiquité : l'Europe et tout le pourtour du bassin méditerranéen. Notons du reste qu'avec le développement de programmes transatlantiques sur les colonies françaises du Nouveau Monde, par exemple, ce cadre géographique est encore appelé à évoluer.

Cet espace de travail, si large soit-il, ne doit pas être confondu avec un projet d'inventaire exhaustif. Comme par le passé, les objets inventoriés dans Artefacts ne peuvent être enregistrés que dans le cadre d'un projet scientifique. C'est bien ce projet qui définit le choix du territoire de réflexion, auquel correspond visuellement un fond de carte adapté.

Au sein de Syslat ${ }^{\circledR}$, Dicobj a d'abord existé sous divers formats (FileMaker, Hypercard...) avant de devenir une base de données MySQL. Le projet, qui regroupait environ 30000 objets en 2012, a changé de nature, d'envergure et bien sûr d'objectifs en passant à cette date sur les serveurs de 
l'Université Lumière Lyon 2. L'investissement des étudiants (master et doctorat) rattachés à l'UMR 5138, Archéologie et Archéométrie, a été essentiel dans cette mue conceptuelle et technique. Signalons enfin que Dicobj, dans sa conception initiale, a été entièrement repris par M. Py qui en a publié quelques années plus tard une version en ligne, ainsi qu'un ouvrage papier (M. Py, 2016) ultérieurement mis en ligne, achevant ainsi, pour les âges du Fer, la trilogie Dicocer, Dicomon et Dicobj qu'il avait imaginée en reprenant la fouille de Lattes au début des années 1980.

\section{Architecture}

Artefacts est aujourd'hui une base de données SQL, interrogée en PHP ou HTML à l'aide d'interfaces en ligne. Le cœur du projet consiste en une base de données dont tous les fichiers communiquent entre eux : une bibliographie, une liste de sites archéologiques, de musées et de personnes, des codes descriptifs et des pages typologiques reliées à une bibliothèque d'images. La mention de sites archéologiques dans les listes d'attestations des pages typologiques permet de générer automatiquement, sur différentes tailles de fonds proposées à l'utilisateur, des cartes de répartition exportables.

Les objets sont classés selon trois types d'informations, regroupées dans un numéro d'inventaire (figure 3) :

- un code de trois lettres, lui-même associé à une triade de catégories : objet, fonction, domaine ;

- un numéro de période, selon le code suivant ${ }^{1}$ :

- 0 : Néolithique / Chalcolithique (jusque vers -2200);

- 1 : Âge du Bronze (de -2200 à -750)

- 2 : Premier âge du Fer (de -750 à -475)

- 3 : Deuxième âge du Fer (de -475 à -30$)$

-4 : Époque romaine (de -30 à +500$)$

- 5 : Haut Moyen Âge (de 500 à 800 )

- 6 : Moyen Âge central (de 800 à 1250)

- 7 : Bas Moyen Âge (de 1250 à 1450)

- 8 : Gothique / Moderne (de 1450 à 1600)

- 9 : Proto-industriel (de 1600 à 1914)

- un numéro d'ordre de 4 ou 5 chiffres, attribué à chaque forme de manière incrémentale au moment de la création.

Pour les francophones, l'association d'un code mnémotechnique et d'un identifiant chronologique facilite les recherches généralistes, $\mathrm{HCH}-5$ permettant de retrouver par exemple les haches du haut Moyen Âge. Mais différents menus permettent aussi de retrouver soit les haches de toutes périodes, soit tous les objets du haut Moyen Âge.

Toute recherche effectuée sur le site génère une première sélection de fiches, au sein desquelles on peut ensuite effectuer une autre recherche. Ces requêtes successives imbriquées constituent la manière la plus simple de retrouver, de manière de plus en plus affinée, un objet dans une catégorie donnée.

Les quelques 600 codes actuellement ouverts peuvent regrouper des objets de noms, de formes ou d'époques différentes, pourvu que tous répondent au même classement fonctionnel : les clés de tri

\footnotetext{
${ }^{1}$ En dehors de l'Europe nord-occidentale, les bornes chronologiques sont les seules à avoir du sens, l'attribution culturelle pouvant varier selon les régions. 
fonction et domaine permettent de retrouver, à partir de la page Codes, tous les objets de la catégorie supérieure. Chaque sélection de fiches peut être cartographiée ou, si on le souhaite, faire l'objet d'un document mis en page au format PDF.

Liste des codes (612) :
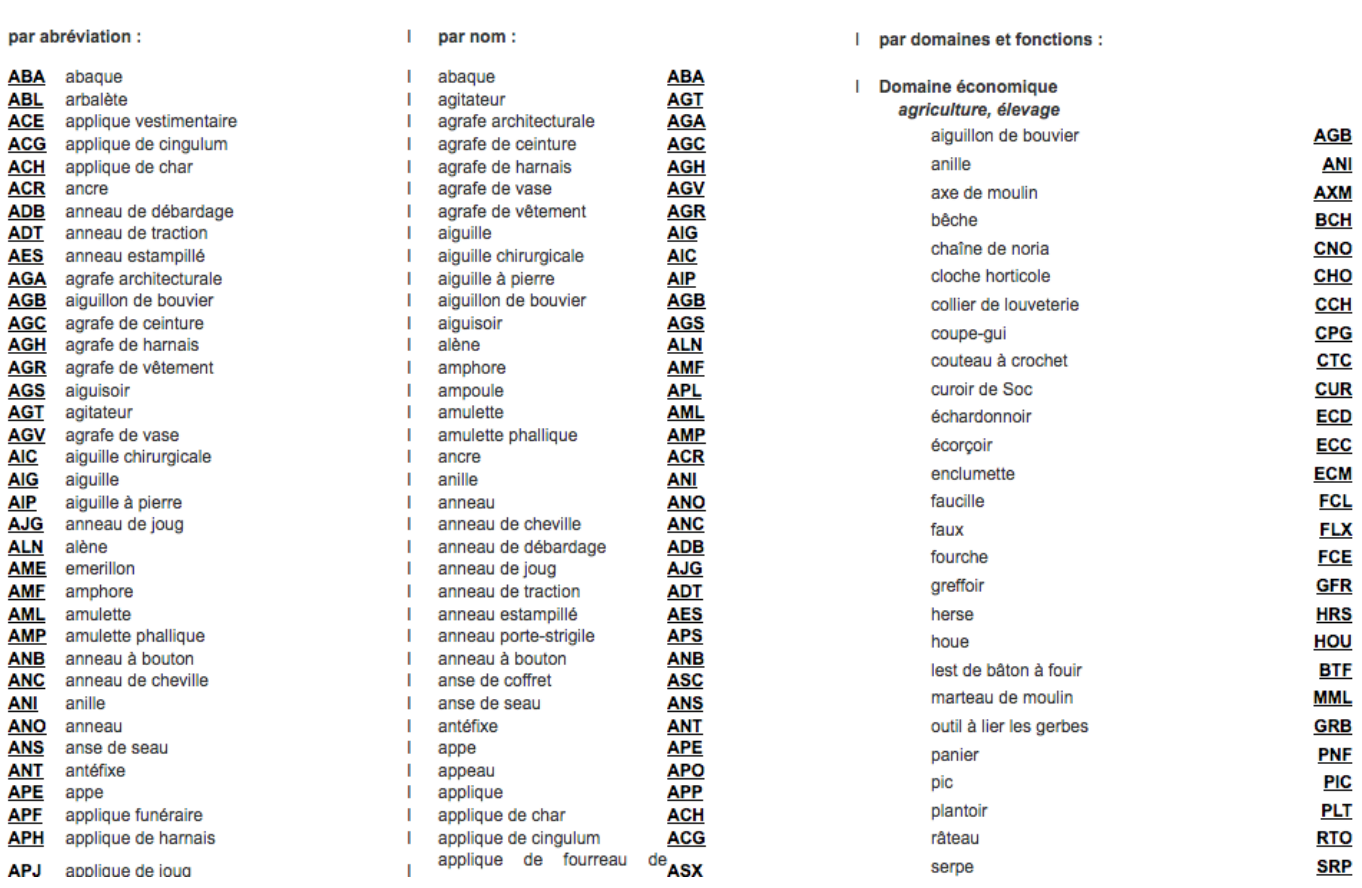

Figure 3. Aperçu de la page Codes.

\section{Usages}

Deux façons d'utiliser Artefacts sont proposées, selon que les utilisateurs souhaitent ou non pouvoir écrire sur les pages du site : visiteur ou membre, puis auteur. Le visiteur accède à la totalité de la bibliographie ainsi qu'à la liste des musées, mais seulement aux vignettes des fíches typologiques définies comme publiques par les auteurs (environ deux tiers, actuellement). Ce niveau qui offre déjà beaucoup de fonctionnalités (plusieurs milliers de publications en ligne, aperçu de dizaines de milliers d'images) s'avère suffisant pour une bonne partie des utilisateurs du site, qui y trouvent une ressource gratuite, facilement accessible et néanmoins très spécialisée.

Les personnes qui souhaitent accéder à plus de données sont invitées à manifester leur engagement de plusieurs façons. Un stade intermédiaire entre visiteur et auteur permet, sur une simple inscription en ligne, de tester l'investissement des personnes motivées par cette démarche, et dont une partie pourra souhaiter s'investir encore davantage. L'inscription ouvre un espace personnel dans lequel sont enregistrées les préférences de l'utilisateur, de même que ses actions sur le site. Il peut ajouter des références à la bibliographie générale, et aussi accéder à un forum interactif dans lequel sont discutées des questions diverses : actualités de la recherche, informations scientifiques, débats, demandes d'identification. À la différence du simple visiteur, le membre inscrit peut visualiser le contenu des fiches publiques, alors que le visiteur était limité à la mosaïque de vignettes donnant un simple aperçu des fiches trouvées.

L'accès au statut d'auteur, à la différence des précédents, doit faire l'objet d'une demande motivée, examinée par les administrateurs du site. Les candidats peuvent proposer des tâches diverses, dont ils conservent évidemment l'initiative et la maîtrise : enregistrer le mobilier d'une fouille, les objets d'un musée ou d'une région donnée ; classer une catégorie de mobilier, compléter les références typologiques d'une série ou même proposer un nouveau système... Compte tenu de la variété des aires géographiques ou chronologiques couvertes par Artefacts, chaque spécialité peut avoir des propositions 
spécifiques. L'auteur validé peut voir la totalité des fiches du site, publiques ou non ; écrire sur les fiches existantes ; en créer ou même en supprimer.

D'une manière plus générale, la première tâche, qui est loin d'être achevée, consiste à ouvrir une fiche par forme dans chaque catégorie de mobilier (figure 4) : cela a été fait par exemple, ces dernières années, pour la verrerie antique, médiévale et moderne, pour les fibules ou pour d'autres séries. Une fois le cadre typologique tracé, chaque fiche doit être alimentée avec des attestations confirmées, indiquant dans chaque cas le contexte, la référence de fouille et la datation, le lieu de conservation et la bibliographie. Cette étape particulièrement ambitieuse ne sera bien sûr jamais achevée en raison du renouvellement constant des données archéologiques. Elle s'efforce seulement d'indiquer à l'utilisateur quelques exemples représentatifs et, si possible, d'esquisser la répartition du type dans son espace chrono-culturel (figure 5). Les données proviennent principalement du dépouillement de la bibliographie, mais nous y ajoutons toutes les fois que c'est possible des documents inédits provenant de fouilles ou de collections muséales.

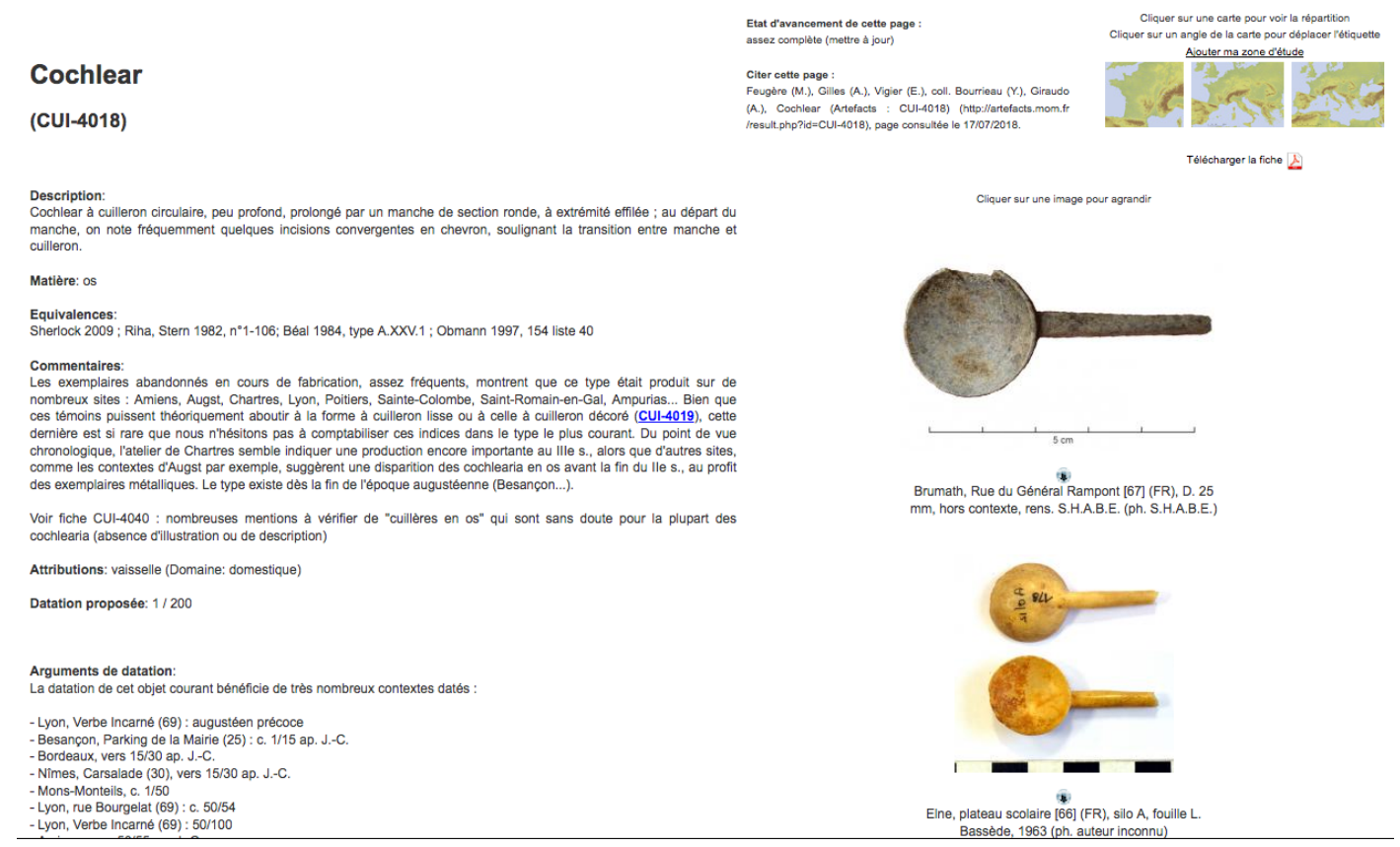

Figure 4. Aperçu de la fiche typologique des cochlearia en os (CUI-4018).

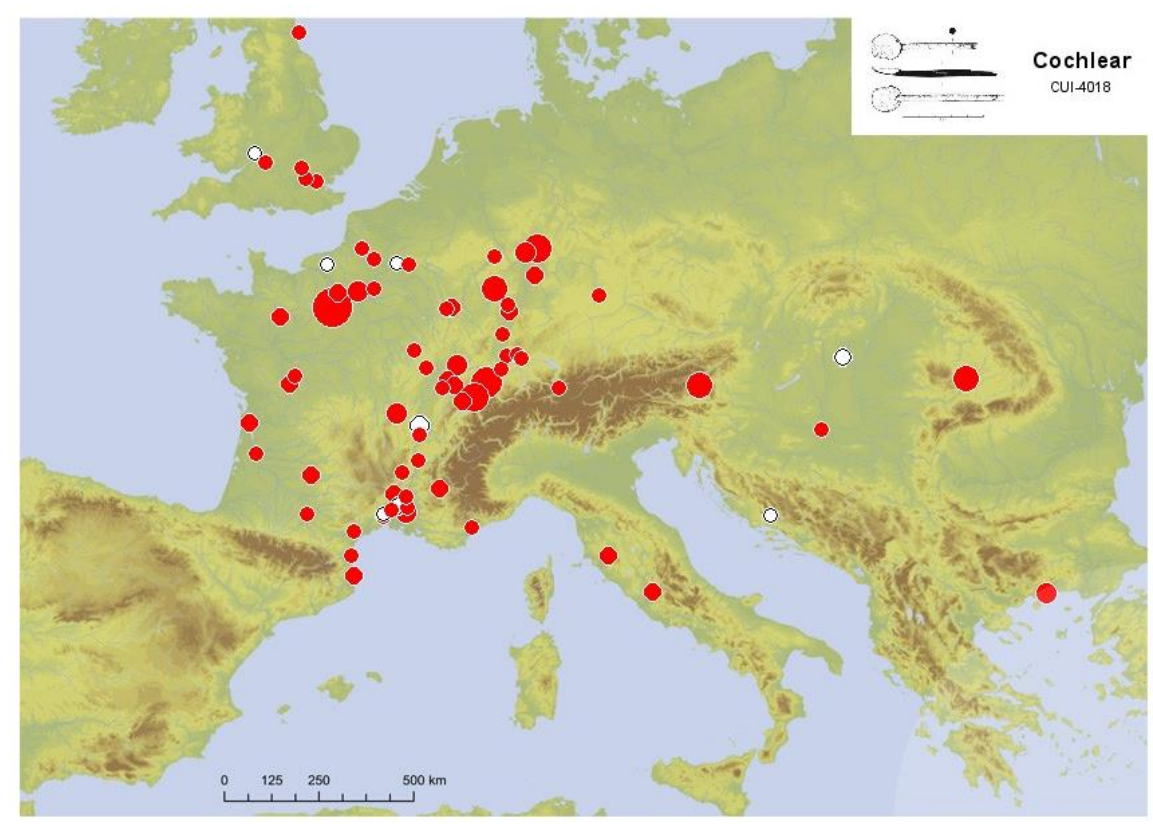

Figure 5. Carte de répartition de CUI-4018. 


\section{Saisie}

Les données enregistrées dans Artefacts proviennent principalement de deux sources : les dépouillements bibliographiques et la saisie primaire à partir de mobiliers archéologiques. Les dépouillements sont effectués soit par des équipes universitaires qui répertorient toute la documentation publiée, par exemple, dans une revue. Tous les articles consacrés à (ou contenant) des objets archéologiques, sont enregistrés, et quand c'est possible, leur contenu est analysé et ajouté aux pages concernées. D'autres enregistrements sont effectués par des chercheurs qui, à partir de leurs propres inventaires, ou de listes publiées par ailleurs, alimentent les pages typologiques.

L'enregistrement primaire est effectué lui aussi, en général, par des étudiants accédant à du mobilier des collections publiques dans le cadre de leurs recherches personnelles. Les équipes d'Artefacts ont organisé des missions dans divers musées (Saint-Romain-en-Gal, Lyon, Besançon, Biesheim, Aquileia...) et une mission récurrente, au Musée Archéologique d'Ampurias (Espagne), permet depuis quelques années à un groupe d'une dizaine de chercheurs de se former aux techniques d'inventaire, de photographie et de classement typologique (Feugère, 2016). Quelques conservateurs de musées sont également engagés dans une démarche d'inventaire et de classement de leurs collections, Artefacts servant alors de cadre de référence typo-chronologique.

\section{Recherche}

On le voit, au-delà du simple regroupement de la documentation et de son classement, l'image d'un type archéologique qui apparaît finalement dans Artefacts constitue en elle-même un nouvel objet scientifique. La proportion des formes archéologiques bien étudiées, avec des listes suffisamment complètes et fiables, est naturellement réduite. De très nombreux types d'objets sont inédits et davantage encore, sans être complètement inconnus, n'ont jamais bénéficié d'une attention particulière de la part des spécialistes. Ce sont même, de façon paradoxale mais finalement bien compréhensible, les formes d'objets les plus communes qui sont les moins étudiées et donc les moins connues. En offrant aux archéologues des listes complétées par plusieurs dizaines d'auteurs, Artefacts contribue à élaborer, notamment sur ces formes très abondantes, des données nouvelles que chacun peut compléter ensuite avec ses données locales, entrant dans un rapport gagnant-gagnant où chaque contributeur fait progresser la connaissance commune, tout en bénéficiant des apports de ses prédécesseurs. Cette mutualisation des données documentaires est une réelle nouveauté dans l'histoire de la discipline (Feugère, 2011 ; 2015) et se heurte aux approches académiques traditionnelles par nature très individualistes puisque fortement liées à la notion de "propriété intellectuelle ». Cette étape s'avère néanmoins nécessaire pour faire émerger des réflexions fondées sur des bases scientifiques solides puisqu'elle seule permet de s'affranchir, pour partie, de la collecte des données, tâche devenant de plus en plus accablante au fil des découvertes. Quelques exemples, dont beaucoup proviennent des travaux de doctorants dont la thèse a largement utilisé Artefacts, permettront d'illustrer cette démarche.

Le simple état de la documentation permet parfois de justifier telle ou telle étude. Ainsi, une équipe de chercheurs dirigée par J.-P. Girault commence-t-elle par montrer la carte de toutes les fibules étudiées en France pour montrer le vide de la région envisagée et donc la nécessité d'un inventaire des fibules du Lot (Girault et al., 2016). Pour étudier les fibules émaillées de Gaule Belgique et de Germanie Inférieure, M. Callewaert a pris en compte l'ensemble des formes connues de ces objets, les a classées et en a dressé des listes permettant d'établir des cartes de répartition par type, mais aussi par groupes et par styles (Université Libre de Bruxelles, 2016). Plus simplement, les auteurs qui publient des séries régionales prennent l'habitude de mentionner systématiquement les numéros d'inventaires des types Artefacts pour que le lecteur puisse se reporter, s'il le souhaite, à des données plus complètes sur chaque forme (Abaz, Storai 2018).

L'étude des militaria de Lyon-Lugdunum a amené L. Guillaud (2017) à rassembler toute la documentation connue sur les types attestés dans son corpus : il en a critiqué la typo-chronologie, a 
rassemblé et complété les inventaires, pour en tirer des cartes mises à jour à partir desquelles il discute l'origine des troupes en présence et l'évolution de leur recrutement, suggéré notamment par les cartes de répartition de chaque élément. De son côté, E. Vigier qui étudie les objets liés à l'hygiène et à la médecine en Gaule, a répertorié l'ensemble des objets de toilette et des instruments à usage chirurgical ou pharmacologique sur son territoire d'étude. Là encore, la démarche implique un inventaire, un classement et une réflexion typo-chronologique sur des données très dispersées, qu'il faut organiser avant de pouvoir en discuter la signification (Université Lumière Lyon 2, en phase d'achèvement en 2018).

Les études spécialisées, impliquant l'inventaire raisonné d'importantes séries de documents mobiliers, sont évidemment les premières à trouver dans Artefacts l'outil idéal ; mais des démarches moins généralistes, par exemple des sujets régionaux voire locaux, peuvent également y trouver leur compte. Ainsi, pour étudier la culture matérielle des populations occupant le territoire de la colonie de Valence, A. Gilles (2016) a utilisé Artefacts pour insérer sa documentation dans un contexte élargi, ce qui lui a permis de mieux caractériser les données et d'en discuter la signification sur le territoire étudié. L'étude des militaria tardifs de la ville romaine d'Aquileia, en Vénétie, a elle aussi bénéficié de classements, d'inventaires et de cartes systématiquement réalisés avec Artefacts (Feugère, 2012).

Une démarche similaire est actuellement en cours sur les verreries tardo-médiévales et modernes (A. Berthon). Des pans entiers de la documentation, les parures carolingiennes par exemple, commencent à apparaître comme un ensemble homogène grâce aux inventaires constitués dans Artefacts (A. Berthon, M. Feugère). A la différence d'une publication traditionnelle, les progrès sont ici diffus et pratiquement continus, et il peut sembler difficile de les appréhender. Les spécialistes voient pourtant clairement l'évolution de la situation documentaire et le progrès des connaissances. Les publications disponibles sur ces parures ont d'abord été enregistrées puis dépouillées dans Artefacts, apportant une base solide sur les fibules émaillées, par exemple. Sur la base de ces travaux, on pouvait croire que les fibules émaillées carolingiennes, par exemple, se limitaient à une grande inter-région centrée sur l'ouest de l'Allemagne, la Belgique, les Pays-Bas et la Scandinavie. Mais la mise à plat de ces classements permet désormais aux archéologues français de reconnaître et de publier leurs propres découvertes, parfois mal identifiées. De nouvelles catégories de parures, les agrafes bimétalliques, généralement coulées dans un alliage de plomb-étain sur une aiguille en fer, ont pu être mises en évidence et documentées à partir de découvertes françaises. Des formes d'agrafes jusque-là surtout connues en Grande-Bretagne (tag-hooks) s'avèrent également attestées en Gaule et sur la péninsule ibérique, avec dans les régions méditerranéennes des formes jusqu'ici non reconnues. On peut citer un autre exemple avec la catégorie plus anciennement décrite mais insuffisamment étudiée des agrafes à double crochet. La forme sans doute la mieux représentée en France pour cette période, fait désormais l'objet de vastes inventaires, base d'études affinées. C'est dire que tout un pan de la recherche, autrefois presque inconnu, se dévoile et se structure aujourd'hui dans tout un pays grâce à Artefacts.

Avec son développement principalement européen à ce jour, Artefacts peut enfin apporter une base solide à des projets novateurs : ainsi, l'installation des premiers Français dans les colonies d'Amérique à travers l'importation d'objets métalliques, de perles en verre ou encore de la vaisselle en verre, sont susceptibles de mettre en évidence des flux mal connus ; c'est en tous cas l'objectif du projet transatlantique dirigé par A. Bonneau et J. Soulat (GRAUL-Université Laval de Québec, et CRAHAM UMR 6273 - Université de Caen), auquel Artefacts est associé : des fonds de cartes montrant la répartition d'un même type sur les deux rives de l'Atlantique pourront montrer à quel réseau de production se connectent les colons des XVII ${ }^{\mathrm{e}}$ et XVIII ${ }^{\mathrm{e}}$ siècles.

D'un point de vue plus général, l'expérience des auteurs d'Artefacts dans la dénomination, la définition et le classement des types d'objets leur a permis de mettre en place, avec le Labex IMU (Intelligence des Mondes Urbains, Lyon), un programme de définition d'un thésaurus des objets archéologiques, qui sera dirigé puis intégré à la gestion des requêtes informatiques grâce à deux contrats post-doctoraux, en 2019-2020. Cette action de recherche spécifique, qui sera dirigée par 
l'équipe d'Artefacts mais fera largement appel au travail collaboratif, bénéficiera à terme à l'ensemble de la communauté archéologique. Elle fera l'objet de séminaires et de publications spécifiques afin de garantir à la fois sa fiabilité et sa visibilité.

$* * *$

Il est clair que les applications d'Artefacts apparaissent et apparaîtront au fur et à mesure du développement de la base.

Certaines d'entre elles sont purement documentaires : la richesse de la bibliographie, par exemple, amène de plus en plus d'utilisateurs. Mais ce n'est pas seulement l'abondance des données, pourtant en progression constante, qui fait l'intérêt d'Artefacts. C'est surtout leur classement, opéré et vérifié en permanence par des chercheurs qualifiés, qui fait l'intérêt de cette documentation, à l'inverse d'une acquisition automatique des données à l'instar de nombreuses autres bases de données. Rien n'entre dans Artefacts sans avoir été analysé et ordonné par une personne compétente et bien identifiée. De ce fait, le projet offre un surplus qualitatif aux bases de données qui regroupent, par exemple, les seules données d'un type particulier, comme la bibliographie. Grâce à Artefacts, on peut par exemple connaître (éditer, visionner, trier, observer sur une carte...) le détail des données apparaissant dans une publication. Cette valeur ajoutée, étroitement liée à l'architecture d'Artefacts, nous permet d'envisager pour l'avenir des partenariats fructueux avec les différents projets qui fleurissent un peu partout en Europe sur le mobilier archéologique. Nul doute que ces partenariats nous réservent pour les années à venir de nouvelles opportunités et surprises scientifiques.

\section{Bibliographie sur Artefacts}

FEUGÈRE, Michel 2010 : « The Artefacts Project : An Encyclopaedia of Archaeological Small Finds ». Lucerna (The Roman Finds Group Newletter) n³9, Sept. 2010, 4-6.

http://artefacts.mom.fr/Publis/Lucerna.pdf

FEUGERE, Michel 2011, « Artefacts : encyclopédie en projet, outil d'aujourd'hui ». Instrumentum n³3, juin 2011, 24-27.

https://www.academia.edu/1174934/Artefacts encyclopédie en projet outil daujourdhui

FEUGERE, Michel 2015, « Bases de données en archéologie : de la révolution informatique au changement de paradigme ». Cahiers Philosophiques n ${ }^{\circ} 41,2015,139-147$.

https://halshs.archives-ouvertes.fr/halshs-01242413

FEUGERE, Michel 2016, « Artefacts en 2015-2016 ». Instrumentum 43, juin 2016, 54-57.

https://www.academia.edu/26649161/Artefacts_en_2015-2016._Instrumentum_43_juin_2016_54-57

FEUGÈRE, Michel, BULTincK, Patrick 2015, « Artefacts, dé bron voor alles wat u wou weten over kleine archeologische objecten, wil Vlaanderen op de kaart zetten ». www.Archeonet.be, 30 mar 2015.

http://www.archeonet.be

http://www.archeonet.be/images/artefacts.pdf

Feugere et al. 2017 : M. Feugère, E. Vigier, L. Eyango, A. Giraudo, Artefacts v2 : les enjeux d'une migration. HALSHS, 18/06/2017.

https://halshs.archives-ouvertes.fr/halshs-01541204

FEUGERE, Michel, GILLES, Amaury, VIGIER, Elise, à paraître, « L'archéologie face aux objets 》 (à paraître, ArAr, revue électronique en ligne).

\section{Bibliographie citée}

ABAZ, Bernard, StORAI, Romain, 2018, Les fibules du Mas d'Agenais. In: Documents d'Archéologie Lot-et-Garonnaise 2018, 7-26. 
BATS, Michel, et al. 1986, Enregistrer la fouille archéologique: le système élaboré pour le site de Lattes, Hérault, ARALO, série Lattes, 1986.

CALLEWAERT, Maxime, 2016, Les fibules émaillées en Gaule Belgique et Germanie Inférieure : chronologie, production et consommation (Thèse de doctorat de l'Université Libre de Bruxelles), Bruxelles, 2016.

Feugere, Michel, 2012, « Tra Costantino e Teodosio (IV-V secolo d.C.). Osservazioni sui militaria di Aquileia ». Aquileia Nostra 83-84, 2012-2013, 317-344.

\section{http://artefacts.mom.fr/Publis/Feugere_2012-2013_[Militaria_d_Aquileia_tardifs].pdf}

Feugere, Michel, Py, Michel, 2011, Dictionnaire des monnaies découvertes en Gaule méditerranéenne (530-27 av. notre ère), éd. Monique Mergoil - Bibliothèque nationale de France, Montagnac-Paris.

GILLES, Amaury, 2016, Vivre et produire dans les campagnes de la colonie de Valence (IIe s. av. J.-C. - VIe s. apr. J.-C.), Thèse de doctorat, Université Lyon 2, Lyon 2016.

GIRAULT, Jean-Pierre et al., 2016 : J.-P. GIrault, J.-M. Beausoleil, A. Collet, J. Gascó, J.-P. Guillaumet, L. Izac-Imbert, D. Rigal, M. Vidal, Les fibules dans le département du Lot : catalogue et inventaire. In: Annales des XXIVe Rencontres archéologiques de Saint-Céré (Lot) 2016, 9-62.

Guillaud, Lucas, 2017, Militaria à Lugdunum. Etude de l'armement et de l'équipement militaire d'époque romaine à Lyon (Ier s. av. - Ve s. ap. J.-C.) (Thèse de Doctorat, dir. M. Poux, Université Lumière - Lyon 2), Lyon 2017 [à paraître].

PY, Michel, et al., 1991 : M. Py, J. López, R. Buzo i Capdevilla, A. Adroher Auroux, D. Garcia, P. Weidelt, M. Feugère et coll., Système d'enregistrement, de gestion et d'exploitation de la documentation issue des fouilles de Lattes (Lattara 4), Lattes 1991.

PY, Michel, et al., 1997 : M. Py avec A. Adroher, S. Barberan, R. Buxó, F. Conche, M. Feugère, A. Gardeisen, C. Jandot, J. López, V. Mathieu, M. Monteil, H. Pomarèdes, St. Raux, V. Rinalducci, Fr. Souq, Syslat 3.1. Système d’information Archéologique. Manuel de référence (Lattara 10), Lattes 1997.

Py, Michel, 1993 (dir.), Dictionnaire des céramiques antiques (VIIe s. av. n. è. - VIIe s. de n. è.) en Méditerranée nordoccidentale (Provence, Languedoc, Ampurdan) (Lattara 6), Lattes 1993.

PY, Michel, 2006, Les monnaies préaugustéennes de Lattes et la circulation monétaire protohistorique en Gaule méridionale (Lattara 19), 2 vol., Lattes 2006.

PY, Michel, 2016, Dictionnaire des objets protohistoriques de Gaule méditerranéenne (IXe - Ier siècles avant notre ère) (Lattara 23), Lattes 2016.

\section{http://cchum-kvm-syslat.in2p3.fr//SLC/DICOBJ/d.index.html}

SOULAT, Jean, BONNEAU, Adelphine, en préparation. Le petit mobilier dans les colonies françaises d'Amérique aux XVI $X I X^{e}$ siècle. Réflexions sur la diffusion transatlantique de la culture matérielle française, Archéologie Moderne et Contemporaine, 6, Editions Mergoil, en préparation.

VIGIER, Elise, à paraître, L'instrumentum d'hygiène et de médecine en Gaule romaine (Thèse, Univ. Lyon 2 et Lyon 3, M. Poux et B. Cabouret dir.), en préparation. 